\title{
L'eau de boisson : le juste apport
}

I y a environ 3,5 milliards d'années la vie est apparue dans l'eau. Puis, il y a 500 millions d'années la vie est sortie de

l'eau pour gagner le milieu terrestre, mais l'eau doit toujours constituer le composant essentiel du milieu intérieur hors duquel nulle cellule, particulièrement nulle cellule humaine, ne peut survivre. De plus, l'eau est le principal constituant de ces mêmes cellules. Il est remarquable que tous les articles scientifiques présentés dans ce numéro de Médecine \& nutrition montrent combien l'état hydrominéral des êtres vivants supérieurs, dont l'Homme, est tout à la fois finement régulé par la mise en jeu de multiples systèmes de défense, et fragile donc à grand risque de perturbation. Tous soulignent l'importance d'un bon état d'hydratation pour le maintien de fonctions physiologiques optimales, tant métaboliques que physiques et cognitives.

Mais qu'est-ce qu'un bon état d'hydratation ? Il est bien établi que le métabolisme de l'eau est étroitement associé à celui des minéraux osmotiquement actifs, principalement (mais non exclusivement) celui du sodium. Ne parle-t-on pas d'ailleurs de métabolisme hydrominéral ? Le mot déshydratation décrit génériquement toutes les situations pathologiques où les grandeurs caractéristiques de ce métabolisme hydrominéral s'écartent mesurablement de leur valeur normale, tant parce que le contenu en eau ou celui en électrolytes est perturbé. Ces situations sont généralement graves et sources de nombreux dysfonctionnements qui parfois peuvent même rapidement entraîner la mort. Mais toutes les contributions à ce numéro spécial nous apprennent qu'avant d'atteindre un état de désordre hydro-électrolytique aussi dramatique, il existe de très fréquentes situations beaucoup plus insidieuses, caractérisées par un habituel déficit d'apport en eau, moindre mais occulte, et dont les conséquences à long terme peuvent être tout aussi néfastes.

Pourtant, dans ces cas, grâce à la grande efficacité des systèmes de régulation de l'équilibre osmotique, la natrémie et la pression osmotique du milieu intérieur apparaissent normales en dépit d'un déficit global de l'organisme en eau. Comment qualifier de tels états pour lesquels le mot déshydratation est sans doute trop fort ? Peut-être hypohydratation, mais ce mot n'est-il pas trop proche du précédent ? Dans ces cas, il semble que ce soit d'abord le volume total d'eau de l'organisme qui soit amoindri. Force est de constater que la science n'a pas inventé un terme spécifique pour décrire une telle situation d'« hypovolhydrie » (ou d'infravolhydrie), sans doute parce que l'on ne sait pas bien la caractériser, ni en mesurer l'ampleur dans les conditions courantes. La mesure de l'eau totale d'un être vivant nécessite en effet de déployer des techniques très sophistiquées, utilisant de coûteux isotopes stables (de l'eau doublement marquée) et des techniques analytiques que très peu de laboratoires maîtrisent (la spectrométrie de masse isotopique). Un tel état sub-normal, renforcé par l'absence bien connue de réserves hydriques internes mobilisables en cas de besoin, constitue une situation précaire, augmentant le risque de basculer dans la déshydratation vraie en cas de conditions environnementales défavorables. Les articles respectivement de Monique Ferry et de Florence Constant soulignent combien ce risque est grand aux deux extrémités de la vie, pour les seniors dont la teneur corporelle en eau décroît avec l'âge, et pour les nourrissons dont la capacité rénale de réabsorption de l'eau est moindre qu'à l'âge adulte.

L'évidente dissociation entre un rigoureux ajustement de l'osmolarité du milieu intérieur (de la natrémie) à sa « valeur normale », et un volume d'eau total pouvant être amoindri, interroge le physiologiste. Il pose la récurrente question de la nature exacte de la grandeur régulée dans le cadre de cette régulation physiologique du métabolisme hydrominéral : est-ce la quantité d'eau totale contenue dans l'organisme, est-ce la charge osmolaire (la natrémie), ou

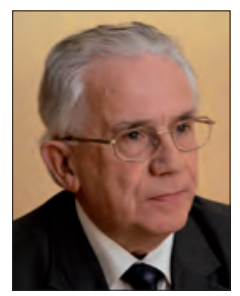

Pr Marc Fantino

Chef du Département de physiologie humaine et nutrition,

Faculté de médecine de l'Université de Bourgogne

et Centre Hospitalier Universitaire (CHU) de Dijon 
les deux ? Physiologiquement, la caractérisation de la grandeur régulée repose sur l'identification des récepteurs permettant sa mesure in vivo. Or, comme l'explique remarquablement Simon Thornton, nous disposons de récepteurs permettant aussi bien la mesure du volume des liquides extracellulaires (indirectement au travers du volume et de la pression du sang), que celle de l'osmolarité du milieu intérieur. Mais toute régulation ne peut être mise en jeu en l'absence d'un signal d'erreur et parfois ne s'enclenche qu'au-delà d'un certain seuil. L'habituel retard d'apparition de la soif, même chez le sujet normal mais plus particulièrement chez le jeune enfant ou chez la personne âgée, peut résulter d'un tel phénomène. Il apparaît de plus que la régulation hydrominérale peut s'adapter, et fonctionner à différents niveaux d'apports hydriques, grâce aux adaptations hormonales. Tout un arsenal neuroendocrinien impliquant d'abord la vasopressine et l'aldostérone, mais aussi le système rénine-angiotensine et d'autres, assure en effet le rétablissement d'une natrémie normale malgré des apports hydriques réduits. Ce processus permet évidemment une adaptation à la pénurie d'eau. Cependant, aussi efficace qu'elle soit, une sur-mobilisation hormonale peut retentir négativement sur de nombreux organes, conséquence des autres effets des hormones mobilisées. L'excès d'angiotensine et d'hormone antidiurétique retentira sur les vaisseaux sanguins, favorisant l'hypertension ; l'augmentation du travail rénal accélérera le vieillissement de cet organe comme l'explique remarquablement Lise Bankir ; Liliana Jimenez rapporte un travail récent montrant qu'une insuffisance de l'apport hydrique entraîne une augmentation de la concentration plasmatique non seulement de I'ADH mais aussi du cortisol avec toutes les conséquences néfastes qui peuvent en résulter.

Aussi, puisque nous avons la chance de vivre dans un environnement où les sources d'eau potable abondent, est-il physiologiquement et médicalement souhaitable de faire habituellement fonctionner notre régulation hydrominérale au niveau optimal de remplissage hydrique de l'organisme ? Cela suppose des apports hydriques quotidiens significativement plus élevés que ceux indiqués par les récentes enquêtes de consommation hydrique. En effet, les études de consommation rapportées par les diverses contributions à ce numéro spécial de Médecine \& nutrition révèlent que les situations d'hypo-consommation sont extrêmement fréquentes. Aussi est-il important que chacun prenne conscience du niveau réel de ses apports hydriques quotidiens et que les médecins traitants, lorsqu'ils interrogent leurs patients sur leur régime alimentaire, n'oublient pas d'également leur demander combien ils boivent (et bien sûr, ce qu'ils boivent !).

Mais quel volume d'eau devrait-on consommer tous les jours pour garantir un état physiologique et de santé optimal ? La soif n'étant qu'un signal tardif, témoin d'un déficit hydrique déjà établi, on ne peut pas seulement se fier à cette sensation pour régler ses apports en eau. Dès lors, il convient que chacun soit attentif à la quantité d'eau qu'il consomme réellement chaque jour pour atteindre des apports adéquats. Il est vrai que les apports devant corriger les pertes, qui sont éminemment variables selon les conditions environnementales et de vie de chacun, des repères de consommation précis, uniques pour tous, ne peuvent être fixés. De plus les apports hydriques doivent être significativement accrus dans certains états physiologiques tels que la grossesse ou l'allaitement comme le rappelle Frédérique Teurnier. Cependant, des repères moyens seraient utiles mais manquent cruellement dans notre pays. Jusqu'alors seule l'EFSA (I'Autorité européenne de sécurité des aliments) a proposé des repères d'apports totaux en eau valables pour les hommes et pour les femmes de tout âge, incluant l'eau procurée par les aliments. On ne peut donc que déplorer que le Programme National Nutrition Santé (PNNS), pourtant si prolixe en conseils nutritionnels, limite à ce sujet sa communication à l'indigent repère : « de l'eau à volonté » sans le décliner en conseil plus précis. Il est regrettable que le PNNS ne soit pas plus insistant sur la nécessité d'apports hydriques au-delà de ce que réclame la soif ordinaire.

Il est bien possible que quelques esprits chagrins s'inquiètent des risques d'une éventuelle surconsommation de boissons. Mais s'agissant de boissons uniquement composées d'eau pure, des repères de consommation valables pour la population générale a priori bien portante, dont la fonction rénale est par définition normale, un apport d'eau éventuellement un peu excessif constituerait-il un réel danger? Le risque de surcharge pondérale ne serait-il pas plus grand si ces mêmes sujets devaient consommer tous les jours tous les aliments recommandés par le PNNS ? 